\title{
Parameter Estimation Techniques for Helicopter Dynamic Modelling
}

\author{
Ping Li, Ian Postlethwaite and Matthew Turner
}

\begin{abstract}
This paper investigates the problem of estimating parameters in a state-space helicopter dynamical model for helicopter controller design and evaluation. Two methods, PEM (the prediction error method) and RBPF (the RaoBlackwellized particle filter-based method), are presented in the paper. Computer simulations are carried out to assess and compare the performance of the two methods with different assumptions on the initial conditions of the parameters to be estimated. Based on this assessment, an estimation scheme that combines the two methods is proposed. The proposed scheme is then applied to real data from EH101 helicopter flight tests and a vertical model including rotor coning dynamics is identified.
\end{abstract}

Keywords: Parameter estimation; State-space model; Rao-Blackwellized particle filter; Prediction error method; Helicopter dynamic modelling.

\section{INTRODUCTION}

A prerequisite for helicopter controller design and evaluation is an appropriate mathematical model. Unlike fixedwing aircraft, the dynamic behaviour of a helicopter is very complex with strong fuselage-rotor coupling and inherent nonlinearities. To develop rational explanations for the dynamic behaviour of a helicopter and to facilitate flight control system design, it is often necessary to use a simplified model that is capable of capturing the main characteristics of the system's dynamic behaviour. This is achieved by linearization with small perturbation theory [1]. It essentially amounts to neglecting all except the linear terms in the Taylor series expansions of the nonlinear model function about the known trim point. The resulting linearized helicopter dynamic model is of the following state-space form:

$$
\dot{\mathbf{x}}=\mathbf{A x}+\mathbf{B u}+\mathbf{w}
$$

where $\mathbf{A}$ and $\mathbf{B}$ are known as the stability and control matrices that are derived from the partial derivatives of the nonlinear model function (see [1]); $\mathbf{x}$ and $\mathbf{u}$ are respectively the perturbed state and input about the known trim point. The term $\mathbf{w}$ represents atmospheric and other disturbances which are assumed to be white Gaussian processes.

There are several approaches to model building. The socalled "white-box" approach starts from first principles and a model is derived from basic physical laws that govern the behaviour of the system. This approach works for simple examples, but its complexity increases rapidly for real-world systems such as the helicopter. The "black-box" approach, sometimes known as system identification, generates a model based entirely on the input/output measurements of the

P. Li, I. Postlethwaite and M. Turner are with the Department of Engineering, University of Leicester, UK pl62@leicester.ac.uk, ixp@leicester.ac.uk, mct6@leicester.ac.uk system without trying to model the internal physical mechanisms of the system. The "grey-box" approach is somewhere between the aforementioned approaches and attempts to combine physical modelling with parameter estimation techniques. In the latter approach, the system's equations are known up to some unknown parameters and the problem of system modelling is transformed into a problem of parameter estimation. In general, a model obtained from a "grey-box" approach has better physical interpretations and explanations when compared with those obtained from a "black-box" approach.

The "grey-box" approach is used in this paper for building helicopter dynamic model (1). The order and structure of the model are first chosen from physical considerations subject to some unknown parameters. Parameter estimation techniques are then used to determine the unknown parameters. The rest of the paper is organized as follows. Section II describes the parameter estimation strategy used and simulation studies are performed in Section III. The results of parameter estimation using real flight test data are presented in Section IV, with concluding remarks in Section V.

\section{PARAmeter estimation StRategy}

The linearized helicopter dynamic model in (1) is in statespace form, it is continuous-time and time-invariant. The aim of modelling is to determine the stability and control matrices $\mathbf{A}$ and $\mathbf{B}$ using the available measurements. The measurements needed for determination of such a model depend on the structure and parameterization of the model. For a helicopter, the measured control inputs will include the main rotor collective, the longitudinal cyclic, the lateral cyclic and tail rotor collective that are collected in vector $\mathbf{u}$ as mentioned above. The measured outputs are a "standard" set of variables that have physical significance, such as: airspeed, linear accelerations, angular information (rates and attitudes) etc (see e.g. [2]). They are either state variables or can be approximated as known linear combinations of the states and inputs, thus the measured output vector can be written as:

$$
\mathbf{y}=\mathbf{C x}+\mathbf{D u}+\mathbf{v}
$$

where $\mathbf{C}$ is the measurement matrix, which is determined by the measured variables and sensor locations, and $\mathbf{v}$ is the measurement noise vector.

To identify model (1) using the aforementioned "greybox" approach, it is assumed that the matrices $\mathbf{A}$ and $\mathbf{B}$ are parameterized by $\boldsymbol{\theta}$ from physical understanding and insight. The measurement model does not usually introduce new unknown parameters to be estimated. To facilitate the presentation, the original physically relevant model (1) and 
(2) is rewritten as follows to show explicitly the dependency on the parameter vector $\boldsymbol{\theta}$ :

$$
\begin{aligned}
\dot{\mathbf{x}} & =\mathbf{A}(\boldsymbol{\theta}) \mathbf{x}+\mathbf{B}(\boldsymbol{\theta}) \mathbf{u}+\mathbf{w} \\
\mathbf{y} & =\mathbf{C}(\boldsymbol{\theta}) \mathbf{x}+\mathbf{D}(\boldsymbol{\theta}) \mathbf{u}+\mathbf{v}
\end{aligned}
$$

For most practical applications, the measurements are sampled-data (i.e. discrete), and the parameter estimation algorithm will then be implemented in the discrete-time domain. Suppose that the input is constant over the sampling interval $T$, then the sampled/discrete version of model (3) and (4) is given as follows (see e.g. [5] [3]):

$$
\begin{aligned}
\mathbf{x}_{k+1} & =\boldsymbol{\Phi}(\boldsymbol{\theta}) \mathbf{x}_{k}+\mathbf{G}(\boldsymbol{\theta}) \mathbf{u}_{k}+\mathbf{w}_{k} \\
\mathbf{y}_{k} & =\mathbf{C}(\boldsymbol{\theta}) \mathbf{x}_{k}+\mathbf{D}(\boldsymbol{\theta}) \mathbf{u}_{k}+\mathbf{v}_{k}
\end{aligned}
$$

where $\boldsymbol{\Phi}(\boldsymbol{\theta})=e^{\mathbf{A}(\boldsymbol{\theta}) T}$ and $\mathbf{G}(\boldsymbol{\theta})=\int_{0}^{T} e^{\mathbf{A}(\boldsymbol{\theta}) \tau} \mathbf{B}(\boldsymbol{\theta}) d \tau ; \mathbf{w}_{k}$, $\mathbf{v}_{k}$ are white Gaussian sequences of appropriate strength and are independent of the input $\mathbf{u}_{k}$. Helicopter dynamic model identification then amounts to the estimation of parameter vector $\boldsymbol{\theta}$ which determines matrices $\boldsymbol{\Phi}, \mathbf{G}$ (thus $\mathbf{A}, \mathbf{B}$ ), $\mathbf{C}$ and $\mathbf{D}$. This can be achieved using the iterative prediction error method (PEM) (see e.g. [3]) or by state-augumentationbased recursive nonlinear filtering techniques (see e.g [4], [6]). These will be briefly outlined next.

\section{A. Prediction error method (PEM) for state-space model identification}

PEM is a conventional model identification technique that has been well documented (see e.g. [3]). It consists of two elements: a parameterization of the model to be identified, and then the determination of the model parameters in such a way that the measurements are "explained" as accurately as possible by the model. This is typically achieved by formulating the estimation problem as an optimization problem, in which the variables are the unknown parameters of the model, the constraints are the model equations and the objective or criterion function is a measure of the deviation between the observations and the predictions obtained from the model (i.e. the prediction-error). To sum up, the parameter estimate is obtained by minimizing the prediction-error, so explaining the name of the method.

Following the above description, the parameter estimate $\hat{\boldsymbol{\theta}}_{N}$ is defined by the following minimization (see e.g. [3]):

$$
\hat{\boldsymbol{\theta}}_{N}=\hat{\boldsymbol{\theta}}_{N}\left(\mathcal{Z}^{N}\right)=\arg \min _{\boldsymbol{\theta} \in \boldsymbol{\Theta}} V_{N}\left(\boldsymbol{\theta}, \mathcal{Z}^{N}\right)
$$

Here the function $V_{N}\left(\boldsymbol{\theta}, \mathcal{Z}^{N}\right)$ is, for a given data set $\mathcal{Z}^{N}=$ $\left\{\mathbf{u}_{k}, \mathbf{y}_{k}, k=1,2, \cdots, N\right\}$, a norm used for measuring the "size" of the prediction-error. It is defined as follows:

$$
V_{N}\left(\boldsymbol{\theta}, \mathcal{Z}^{N}\right)=\frac{1}{N} \sum_{k=1}^{N} l(\varepsilon(k, \boldsymbol{\theta}))
$$

where $l(\cdot)$ is a well-defined scalar-valued positive function and $\varepsilon$ is the prediction-error based on the model which is a function of the model parameter $\boldsymbol{\theta}$. In our helicopter application, the model to be identified is of MIMO statespace form as shown in (5) and (6). The following norm or criterion function is used for measuring the "size" of the prediction-error:

$$
V_{N}\left(\boldsymbol{\theta}, \mathcal{Z}^{N}\right)=\operatorname{det} \sum_{k=1}^{N} \mathbf{e}_{k}(\boldsymbol{\theta}) \mathbf{e}_{k}^{T}(\boldsymbol{\theta})
$$

where the prediction-error vector $\mathbf{e}_{k}(\boldsymbol{\theta})$ is defined as:

$$
\mathbf{e}_{k}(\boldsymbol{\theta})=\mathbf{y}_{k}-\hat{\mathbf{y}}_{k}(\boldsymbol{\theta})
$$

Here $\hat{\mathbf{y}}_{k}(\boldsymbol{\theta})$ represents the prediction based on the model (5) and (6) parameterized by $\boldsymbol{\theta}$ and $\mathbf{y}_{k}$ is the actual measurement. For a given data set $\mathcal{Z}^{N}$, the parameter estimate is then defined by the following minimization of (9):

$$
\hat{\boldsymbol{\theta}}_{N}=\arg \min _{\boldsymbol{\theta} \in \boldsymbol{\Theta}} \operatorname{det} \sum_{k=1}^{N} \mathbf{e}_{k}(\boldsymbol{\theta}) \mathbf{e}_{k}^{T}(\boldsymbol{\theta})
$$

which is the maximum likelihood estimate for Gaussian noise with unknown covariance matrix (see [3]). This way of estimating contains many well known and much used procedures. When the data set is given, the function $V_{N}$ defined by (9) is just an ordinary function of a finite-dimensional real parameter vector $\boldsymbol{\theta}$. Determination of the parameter estimate $\hat{\boldsymbol{\theta}}_{N}$ by (11) therefore amounts to a standard question of nonlinear optimization. In general, $V_{N}$ defined by (9) cannot be minimized by analytical methods and a numerical solution which typically involves a nonlinear iterative search has to be used.

For the parameter estimation problem defined by (11), it is the global minimum that interests us. But the determination of such a global minimum can be difficult for general model parameterizations. The criterion, as a function of the parameter $\boldsymbol{\theta}$ given by (9), can define a complicated surface with many local minima, narrow valleys, and so on. This may require substantial interaction from the user in providing reasonable initial parameter values to start the search so as to avoid being trapped into a local minimum. To find the global solution, there is usually no other way than to start the iterative minimization routine at different feasible initial values and compare the results. For this reason, it is worthwhile to spend some effort on producing good initial values for the iterative search routine. An important option for finding such an initial value is to use some preliminary estimation procedure. For a physically parameterized model structure, as in the present application of helicopter dynamic modelling, it is most natural to use physical insight to provide reasonable initial values.

\section{B. Rao-Blackwellized particle filtering (RBPF)-based method for state-space model identification}

In contrast to the PEM method discussed above, the stateaugumentation-based nonlinear filtering method described next is a recursive (non-iterative) "grey-box" modelling method. Again, the method requires explicit parameterization of matrices $\mathbf{A}$ and $\mathbf{B}$ from physical grounds as in (3).

As an alternative to PEM for dealing with the statespace parameter estimation problem, the parameters to be estimated are viewed as additional states, or more precisely 
we augment the state vector $\mathbf{x}$ with the parameter vector $\boldsymbol{\theta}$ as $\mathbf{z}=\left[\begin{array}{ll}\mathbf{x}^{T} & \boldsymbol{\theta}^{T}\end{array}\right]^{T}$ and re-write the state-space model in terms of the augmented state vector $\mathbf{z}$. We then have the following set of equations (see e.g. [4], [5]):

$$
\begin{aligned}
\mathbf{z}_{k+1}=\left[\begin{array}{c}
\mathbf{x}_{k+1} \\
\boldsymbol{\theta}_{k+1}
\end{array}\right] & =\mathbf{g}\left(\mathbf{z}_{k}\right)+\left[\begin{array}{c}
\mathbf{w}_{k} \\
\mathbf{n}_{k}
\end{array}\right] \\
& =\left[\begin{array}{c}
\boldsymbol{\Phi}\left(\boldsymbol{\theta}_{k}\right) \mathbf{x}_{k}+\mathbf{G}\left(\boldsymbol{\theta}_{k}\right) \mathbf{u}_{k} \\
\boldsymbol{\theta}_{k}
\end{array}\right]+\left[\begin{array}{l}
\mathbf{w}_{k} \\
\mathbf{n}_{k}
\end{array}\right] \\
\mathbf{y}_{k}=\mathbf{h}\left(\mathbf{z}_{k}\right)+\mathbf{v}_{k} & =\mathbf{C}\left(\boldsymbol{\theta}_{k}\right) \mathbf{x}_{k}+\mathbf{D}\left(\boldsymbol{\theta}_{k}\right) \mathbf{u}_{k}+\mathbf{v}_{k}
\end{aligned}
$$

where $\mathbf{n}_{k}$ is white Gaussian noise of appropriate strength introduced for parameter evolution to allow the exploration of the parameter space. Obtaining the parameter estimation recursively consequently becomes a general nonlinear filtering problem. In principle, any available nonlinear filtering algorithm can be used to solve this problem.

Traditionally, the above nonlinear filtering problem of estimating the augmented state $\mathbf{z}$ so as to determine the parameter vector $\boldsymbol{\theta}$ is attacked by the sampled-data extended Kalman filter (EKF) (see e.g. [7], [5], [4]). As indicated in [8], "although this extended Kalman filter approach appears perfectly straightforward, experience has shown that, with the usual state-space model, it does not work well in practice". In fact, there are no results to guarantee the convergence of the resulting EKF in the general case (see [7]). The resulting EKF, while being computationally cheap, is prone to divergence and also the parameter estimates obtained may be sensitive to the choice of initial parameter values (see e.g. [6]). Our early research has also shown that the EKFbased approach is inadequate for the problem of helicopter model identification considered in this paper. For this reason, a newly developed Rao-Blackwellised particle filter (RBPF) based parameter estimation method ([6], [9], see also [10], [11]) is adopted for solving the problem.

1) Preliminaries: The particle filter (also known as the sequential Monte Carlo filter) (see e.g. [12], [13], [14], [15]) is a simulation-based method for general nonlinear nonGaussian state estimation, which attempts to approximate the complete probability density function (pdf) of the state to be estimated. This is in contrast to just estimating the first few central moments, as done for the EKF. The major innovation of the particle filter is to approximate the required, usually complicated, pdf by a swarm of interacting points called "particles" which can be considered as realizations or samples from the required pdf, rather than by a function over the state space. As such, the method is not subject to any linearity and Gaussian constraints on the model. The particle filter will propagate and update these particles as the measurement becomes available and as the number of particles increases, they effectively provide a good approximation to the required pdf. See [16] and [17] for a summary of the state-of-the-art of particle filtering algorithms.

The use of a particle filter for simultaneously estimating the states and parameters in a general nonlinear non-Gaussian state space model has been investigated by a number of authors (see e.g. [18], [19]). The idea is similar to that described above, that is to augment the state vector with unknown parameters, and then to design a particle filter based on the augmented state-space model to perform estimation. A major drawback of such a direct use of a particle filter for parameter estimation is that the large augmented state dimension might be prohibitive for practical use. Also no effort has been made in the above to exploit the analytically tractable substructure in the system. However, when parameter estimation is restricted to linear and Gaussian models, an efficient algorithm can be derived using the so-called RaoBlackwellization/or marginalization technique (see e.g. [14], [16]) which will briefly be explained next.

2) RBPF-based parameter estimation: To estimate $\boldsymbol{\theta}$ in (5) and (6) using the RBPF-based method, the model (12) and (13) for nonlinear filtering is reformulated as follows:

$$
\begin{aligned}
\boldsymbol{\theta}_{k+1} & \sim p\left(\boldsymbol{\theta}_{k+1} \mid \boldsymbol{\theta}_{k}\right) \\
\mathbf{x}_{k+1} & =\boldsymbol{\Phi}\left(\boldsymbol{\theta}_{k}\right) \mathbf{x}_{k}+\mathbf{G}\left(\boldsymbol{\theta}_{k}\right) \mathbf{u}_{k}+\mathbf{w}_{k} \\
\mathbf{y}_{k} & =\mathbf{C}\left(\boldsymbol{\theta}_{k}\right) \mathbf{x}_{k}+\mathbf{D}\left(\boldsymbol{\theta}_{k}\right) \mathbf{u}_{k}+\mathbf{v}_{k}
\end{aligned}
$$

where $p\left(\cdot \mid \boldsymbol{\theta}_{k}\right)$ denotes the pdf given $\boldsymbol{\theta}_{k}$.

The Bayesian approach to estimating the parameters in the augmented system (14) (16) would be to construct the posterior pdf $p\left(\mathbf{z}_{k} \mid \mathcal{Z}^{k}\right)$ of the augmented state $\mathbf{z}_{k}=$ $\left[\begin{array}{ll}\mathbf{x}_{k}^{T} & \boldsymbol{\theta}_{k}^{T}\end{array}\right]^{T}$, and then determine the unknown parameters by estimating the augmented states $\mathbf{z}_{k}$ with $p\left(\mathbf{z}_{k} \mid \mathcal{Z}^{k}\right)$. This results in a nonlinear filtering problem which can be solved by a standard particle filter (see e.g. [12], [13]) based on the augmented model $(14) \sim(16)$. In this case, one has to sample the augmented state vector $\mathbf{z}$ and a standard particle filter will recursively generate a sample-based approximation to the augmented posterior pdf $p\left(\mathbf{z}_{k} \mid \mathcal{Z}^{k}\right)$. The minimum mean squared error (MMSE) estimate of $\mathbf{z}_{k}$ is then calculated using the augmented state samples $\mathbf{z}_{k \mid k-1}(i),(i=1,2, \cdots, m)$ from the standard particle filter as follows:

$\hat{\mathbf{z}}_{k}=E\left[\mathbf{z}_{k} \mid \mathcal{Z}^{k}\right]=\int \mathbf{z}_{k} p\left(\mathbf{z}_{k} \mid \mathcal{Z}^{k}\right) d \mathbf{z}_{k}=\sum_{i=1}^{m} \alpha_{k}(i) \mathbf{z}_{k \mid k-1}(i)$

where $m$ is the number of particles (samples) used in the filter and $\alpha_{k}(i)$ is the probability weight associated with the $i$ th particle at time instant $k$.

However, the MMSE of $\mathbf{z}_{k}$ can be rewritten as:

$$
\begin{aligned}
\hat{\mathbf{z}}_{k} & =\int \mathbf{z}_{k} p\left(\mathbf{z}_{k} \mid \mathcal{Z}^{k}\right) d \mathbf{z}_{k} \\
& =\int\left[\int\left(\mathbf{x}_{k}, \boldsymbol{\theta}_{k}\right) p\left(\mathbf{x}_{k} \mid \boldsymbol{\theta}_{k}, \mathcal{Z}^{k}\right) d \mathbf{x}_{k}\right] p\left(\boldsymbol{\theta}_{k} \mid \mathcal{Z}^{k}\right) d \boldsymbol{\theta}_{k}
\end{aligned}
$$

In the present case, for each given parameter sample, $p\left(\mathbf{x}_{k} \mid \boldsymbol{\theta}_{k}, \mathcal{Z}^{k}\right)$ is Gaussian and the integral in the brackets in (18) can be computed analytically (i.e. the state vector $\mathbf{x}_{k}$ can be marginalised out conditional upon a realisation of $\boldsymbol{\theta}_{k}$ ). This is because, for each realization (or sample) of $\boldsymbol{\theta}_{k}$, we have a single linear Gaussian state-space model as shown in (15) and (16). As such, the required marginalization or Rao-Blackwellization can be carried out exactly using the Kalman filter algorithm. The resulting so called RaoBlackwellized particle filter (RBPF) is similar to the standard particle filter, but we only need to sample the parameter space 
rather than the augmented state space. Thus the size of the space to be sampled is reduced. This will reduce the number of particles (and thus the computation) required or increase the accuracy of the estimates for a given filter complexity. With the RBPF, the pdf $p\left(\mathbf{x}_{k} \mid \boldsymbol{\theta}_{k}, \mathcal{Z}^{k}\right)$ in (18) is given by the Kalman filter, while $p\left(\boldsymbol{\theta}_{k} \mid \mathcal{Z}^{k}\right)$ in (18) is approximated by the particle filter. This will result in each parameter particle being associated with one Kalman filter recursion. It can be thought of as using a particle filter for the nonlinear portion of the problem introduced by augmenting unknown parameters with the state vector, and the Kalman filter for the remainder. Thus the method makes full use of the analytically tractable substructure in the augmented system $(14) \sim(16)$. The details of the algorithm can be found in [6].

3) Practical implementation issues: In the application of the aforementioned RBPF-based parameter estimation approach, it is important to determine the conditional pdf (14), which depends on the method for parameter evolution. A straightforward way is to use a random walk model, $\boldsymbol{\theta}_{k+1}=\boldsymbol{\theta}_{k}+\mathbf{w}_{k}^{\theta}$, where $\mathbf{w}_{k}^{\theta}$ is zero mean Gaussian white noise (see [18], also known as "roughening" noise in [12]), for parameter evolution to introduce artificial dynamics on the parameters to be estimated. Here, the function of the random walk model is twofold: it allows the exploration of the parameter space on the one hand (otherwise, the parameter space would only be explored at the initialization of the algorithm as indicated in [20]) and reduces sample degeneracy in particle filtering on the other. However, the use of independent random shocks in a random walk model for parameter evolution will result in posteriors being far too diffuse relative to the theoretical posteriors for the actual fixed parameters. As such the precision of the resulting estimates is inevitably limited and the estimates may not converge.

The key to yielding converging parameter estimates is to make the variance of the random walk decay with time. To achieve this, kernel smoothing with shrinkage as suggested by Liu \& West in [19] is used for parameter evolution in the present application. With this the evolution of parameter particles is carried out as follows:

$$
\boldsymbol{\theta}_{k+1}=a \boldsymbol{\theta}_{k}+(1-a) \overline{\boldsymbol{\theta}}_{k}+\mathbf{w}_{k}^{\theta}
$$

where $a=(3 \delta-1) /(2 \delta), \delta$ is a discount factor in $(0,1]$ which is chosen as $\delta=0.99$ in the present application; $\overline{\boldsymbol{\theta}}_{k}$ is the Monte Carlo mean of the parameters at time instant $k$ and $\mathbf{w}_{k}^{\theta} \sim \mathcal{N}\left(0, h^{2} \mathbf{V}_{k}\right)$ is the "roughening" noise with $h^{2}=1-a^{2}, \mathbf{V}_{k}$ being the variance matrix of the parameters at time instant $k$. Following the above description, the pdf in (14) is of the following form:

$$
p\left(\boldsymbol{\theta}_{k+1} \mid \boldsymbol{\theta}_{k}\right)=\mathcal{N}\left(a \boldsymbol{\theta}_{k}+(1-a) \overline{\boldsymbol{\theta}}_{k}, h^{2} \mathbf{V}_{k}\right)
$$

Ideally, if the number of particles used for particle filtering tends to infinity, the variance of the "roughening" noise in equation (19) for parameter evolution should decay with time and eventually become close to zero for estimation of the fixed parameters as shown in (20). However, the number of particles used in RBPF will be limited in practice due to the available computing power. For this reason, a lower bound to the variance of the "roughening" noise will need to be set so as to enable the parameter particles to explore a small neighbourhood and avoid pre-maturation of the algorithm caused by the limited number of the particles used. This lower bound may also enable the algorithm to track the slow drifting in the parameters to be estimated as indicated in [6].

\section{APPLICABILITY STUDIES VIA SIMULATIONS}

To study the applicability and to illustrate the operation of the methods described in the last section for helicopter dynamic modelling, the problem of identifying a helicopter vertical dynamic model with flapping (coning) dynamics is considered in this section. The data is generated using a CH-47B research helicopter model taken from [21] where the model is parameterized by $\boldsymbol{\theta}=\left[\begin{array}{llllllll}\theta_{1} & \theta_{2} & \theta_{3} & \theta_{4} & \theta_{5} & \theta_{6} & \theta_{7} & \theta_{8}\end{array}\right]^{T}$ as follows:

$$
\left[\begin{array}{c}
\dot{\beta}_{0} \\
\ddot{\beta}_{0} \\
\dot{w}
\end{array}\right]=\underbrace{\left[\begin{array}{ccc}
0 & 1 & 0 \\
\theta_{1} & \theta_{2} & \theta_{3} \\
\theta_{4} & \theta_{5} & \theta_{6}
\end{array}\right]}_{\mathbf{A}(\boldsymbol{\theta})}\left[\begin{array}{c}
\beta_{0} \\
\dot{\beta}_{0} \\
w
\end{array}\right]+\underbrace{\left[\begin{array}{c}
0 \\
\theta_{7} \\
\theta_{8}
\end{array}\right]}_{\mathbf{B}(\boldsymbol{\theta})} \delta_{0}
$$

where $\beta_{0}$ is rotor flapping angle, $\dot{w}$ is the vertical acceleration of the fuselage and $\delta_{0}$ is the collective input. The true (nominal) parameter values (denoted by $\boldsymbol{\theta}_{n}$ ) used for generating data are given in the first row of Table I. The control input $\left(\delta_{0}\right)$ is chosen as a PRBS signal. To facilitate comparsions, the vertical acceleration $\dot{w}$ and flapping angle $\beta_{0}$ are assumed to be measurable, so the measurement equation is of the following form:

$$
\mathbf{y}=\left[\begin{array}{c}
\beta_{0} \\
\dot{w}
\end{array}\right]=\underbrace{\left[\begin{array}{ccc}
1 & 0 & 0 \\
\theta_{4} & \theta_{5} & \theta_{6}
\end{array}\right]}_{\mathbf{C}(\boldsymbol{\theta})}\left[\begin{array}{c}
\beta_{0} \\
\dot{\beta}_{0} \\
w
\end{array}\right]+\underbrace{\left[\begin{array}{c}
0 \\
\theta_{8}
\end{array}\right]}_{\mathbf{D}(\boldsymbol{\theta})} \delta_{0}+\mathbf{v}
$$

The results of parameter estimation from PEM and RBPFbased methods using different measurements with different assumptions of initial parameter values are summarized in Table I. Two sets of initial parameter values (denoted by $\boldsymbol{\theta}_{0}$ ) have been used with PEM in the simulation studies, i.e. $\boldsymbol{\theta}_{0}=0.8 \times \boldsymbol{\theta}_{n}$ (which indicates good prior knowledge of the parameters to be estimated) and $\boldsymbol{\theta}_{0}=\mathbf{0}$ (which implies poor or no prior knowledge of the parameters to be estimated and simply starts the PEM search from the point $\boldsymbol{\theta}_{0}=\mathbf{0}$ ). It can be seen that, with $\boldsymbol{\theta}_{0}=0.8 \times \boldsymbol{\theta}_{n}$, quite good system eigenvalue estimates $\lambda_{i}(\hat{\mathbf{A}})$ can be obtained from PEM parameter estimation as shown in the second and third rows of the last column in Table I. The parameter estimates obtained with measurements $\dot{w}$ and $\beta_{0}$ being available are also very good, but the parameter estimates are degraded when only $\dot{w}$ is available (this is particularly so for the estimate of $\theta_{6}$ as shown in Table I), which implies that measurements of the rotor degrees of freedom may be required so as to obtain good parameter estimates for the extended model with a particular physically-meaningful parametrization. The parameter estimates and the resulting system eigenvalues obtained from PEM with $\boldsymbol{\theta}_{0}=\mathbf{0}$ are not acceptable as 
TABLE I

PARAMETER ESTIMATES AND THE RESUlting EIGENVALUeS $\lambda_{i}(\hat{\mathbf{A}})$ OBTAINED WITH DiFFERENT METHOdS AND INITIAL PARAMETER VALUES

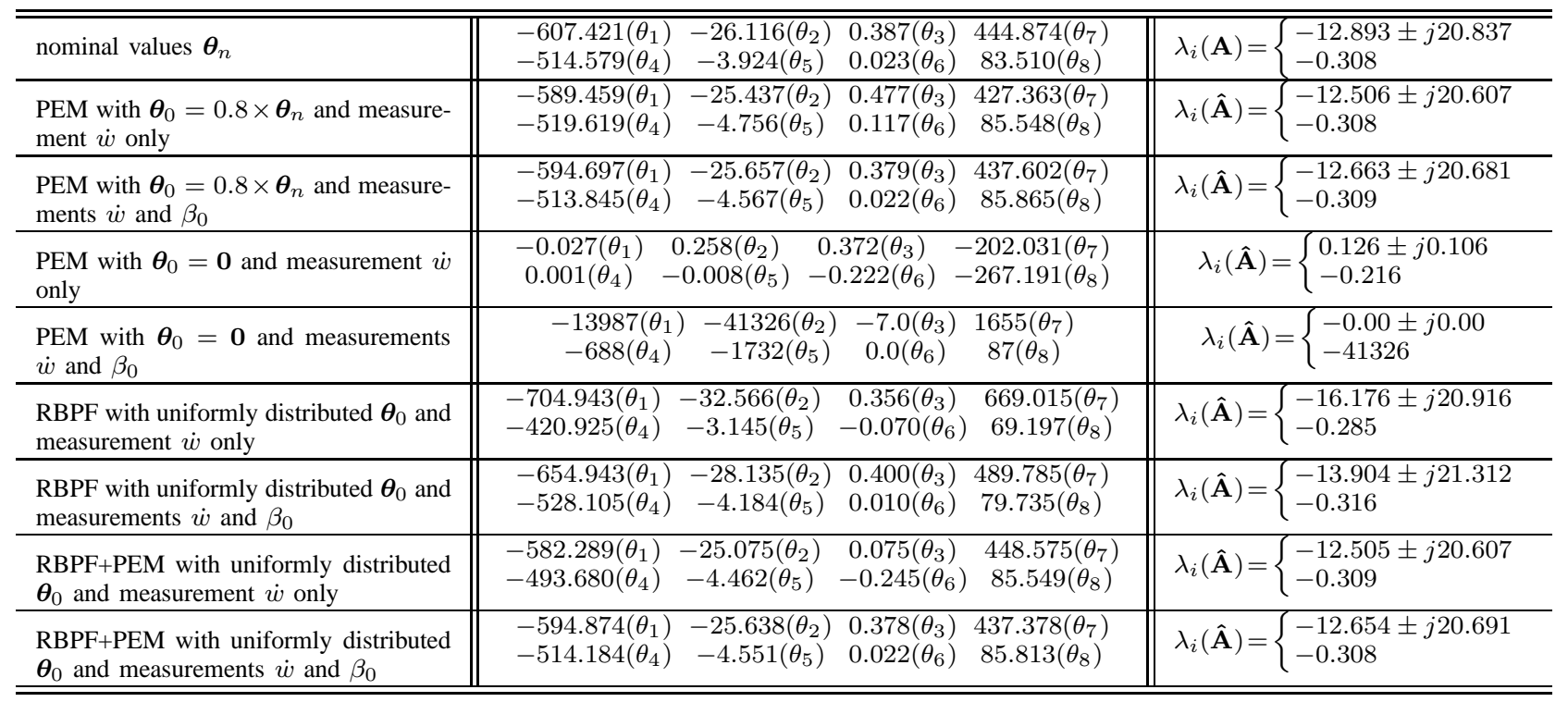

shown in the fourth and fifth rows of Table I, which indicates that the PEM method is sensitive to the choice of initial parameter values. The parameter estimates and the resulting system eigenvalues obtained from RBPF-based method with the number of parameter particles used in the RBPF being chosen as $m=40000$ are shown in the sixth and seventh rows of Table I. The results, though not as good as those obtained from PEM with $\boldsymbol{\theta}_{0}=0.8 \times \boldsymbol{\theta}_{n}$ as shown in the second and third rows of Table I, are acceptable. It needs to be pointed out that these results are obtained with the assumption of very poor knowledge on the initial parameter values. More precisely, the results are obtained with the parameter particles initialized with uniform distributions over the whole possible range of parameter values. As such, the RBPF-based method is much more robust than the PEM method with respect to the choice of the initial parameter values. This motivates the estimation scheme that combines these two methods, i.e. the RBPF-based method is used as a preliminary estimation procedure to produce the initial parameter estimates and PEM is then used to refine the parameter estimates from this initial point. The results of parameter estimation obtained from a combination of these two methods are shown in the last two rows of Table I. Again the parameter estimates are improved when measurement of the rotor degree of freedom $\left(\beta_{0}\right)$ is used as can be seen in the ninth row when compared with the results in the eighth row of Table I.

\section{RESUlTS FROM REAL FLIGHT TEST DATA}

As part of the DARP research project "Towards Robust and Cost Effective Approaches to Rotorcraft Design", the combined estimation scheme proposed in the last section was applied to the identification of a vertical dynamic model for the EH101 helicopter from Westland Helicopters Ltd (WHL) using real flight test data. A segment of data from
EH101 flight tests was selected and the time histories of the measured control input (collective $\delta_{0}$ ) and output (vertical acceleration $\dot{w}$ ) are plotted in Fig. 1 .
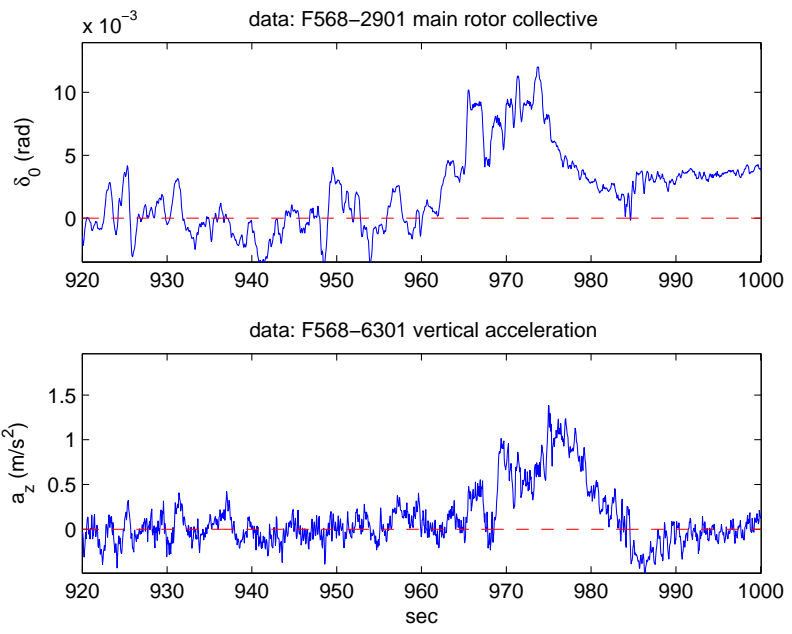

Fig. 1. Flight test data used for identification

The linearised EH101 helicopter vertical dynamic model including coning dynamics is assumed to be of the statespace form of (21). As only vertical acceleration $\dot{w}$ is measured, the measurement equation is given by:

$$
y=\dot{w}=\mathbf{C x}+D u+v=\left[\begin{array}{lll}
\theta_{4} & \theta_{5} & \theta_{6}
\end{array}\right] \mathbf{x}+\theta_{8} \delta_{0}+v
$$

The RBPF-based method is used as a preliminary estimation procedure to generate the initial parameter estimates for PEM and the results are summarized in the first column of Table II. Note that these initial results are obtained with the parameter particles initialized uniformly over the whole possible ranges of the parameter values. The PEM search is then started from 
this initial point and the final parameter estimates and the resulting eigenvalues are given in the second column of Table II.

TABLE II

Parameter estimates and the Resulting eigenValues $\lambda_{i}(\hat{\mathbf{A}})$ OBTAINED WITH EH101 FLIGHT TEST DATA

\begin{tabular}{|c|c|}
\hline$\overline{\overline{\mathrm{RBPF}}}$ & $\overline{\text { RBPF+PEM }}$ \\
\hline$-665.836\left(\theta_{1}\right)-44.172\left(\theta_{2}\right)$ & $-895.455\left(\theta_{1}\right)-30.863\left(\theta_{2}\right)$ \\
\hline $0.210\left(\theta_{3}\right)-126.525\left(\theta_{4}\right)$ & $0.058\left(\theta_{3}\right)-365.428\left(\theta_{4}\right)$ \\
\hline$-0.650\left(\theta_{5}\right)$ & $0.022\left(\theta_{6}\right)$ \\
\hline$-214.169\left(\theta_{7}\right)$ & $-355.872\left(\theta_{7}\right)-85.624\left(\theta_{8}\right)$ \\
\hline$\lambda_{i}(\hat{\mathbf{A}})=\left\{\begin{array}{l}-22.066 \pm j 13.316 \\
-0.026\end{array}\right.$ & $i(\hat{\mathbf{A}})=\left\{\begin{array}{l}-15.420 \pm j 25.630 \\
-0.001\end{array}\right.$ \\
\hline
\end{tabular}

\section{CONCLUding REMARKS}

The problem of estimating parameters in a state-space helicopter dynamic model using time-domain estimation techniques has been studied. Two methods have been presented for solving the problem. Simulation studies have been carried out to assess the performance of the two methods and the main results can be summarized as follows:

- The PEM method can produce good parameter estimates with modest computational demand but needs good initial parameter values to start the search.

- The RBPF-based method can produce reasonable parameter estimates with little prior knowledge of the initial parameter values but is computationally expensive.

- A combination of both methods is believed to be an appropriate solution to the problem considered in this paper. That is, the RBPF-based method is used for producing an initial parameter estimate and PEM search is then started from this initial point to refine the parameter estimate.

The methods presented in this paper require prior knowledge of the particular structure of the model and good initial values /or possible ranges of the parameters to be estimated. If such prior knowledge is not available, "blackbox" system identification techniques (e.g. the subspacebased method) need to be used; see [22] for a recent study. Though reasonable system eigenvalue estimates can be calculated from the results of parameter estimation without using measurements from rotor degrees of freedom, the simulation studies show that such measurements are required to improve the parameter estimates for the extended statespace helicopter dynamic model with a particular physicallyrelevant structure.

The combined estimation scheme proposed in this paper was also applied to real flight test data from WHL and a vertical model including rotor coning dynamics was identified for an EH101 helicopter. Further work is being carried out to validate the identified model.

\section{ACKNOWLEDGEMENTS}

The authors wish to thank Westland Helicopters Ltd (WHL) for provision of the flight test data. Funding support from the UK Department of Trade and Industry (DTI) and
Ministry of Defence (MoD) under the Rotorcraft Aeromechanics DARP Programme is also gratefully acknowledged.

\section{REFERENCES}

[1] Padfield, G. D., (1996). Helicopter Flight Dynamics. Blackwell Science Ltd, Oxford, UK.

[2] AGARD LS-178 (1991) AGARD Lecture Series on Rotorcraft system identification.

[3] Ljung, L. (1999). System Identification-Theory for the User. PrenticeHall, Upper Saddle River, NJ.

[4] Ljung, L., \& Söderström, T. (1983). Theory and Practice of Recursive Identification. The MIT Press, Cambridge, Massachusetts.

[5] Maybeck, P. S. (1982). Stochastic Models, Estimation, and ControlVolume 2. Academic Press, 111 Fifth Avenue, New York.

[6] Li, P., Goodall, R. M., \& Kadirkamanathan, V. (2004). Estimation of parameters in a linear state space model using a Rao-Blackwellised particle filter. IEE Proceedings-Control Theory and Application, 151, No.6, pp727-738.

[7] Ljung, L. (1979). Asymptotic behavior of the extended Kalman filter as a parameter estimator for linear systems IEEE Trans. on Automatic Control, AC-24, No.1, pp36-50.

[8] Anderson, B.D.O., \& Moore, J.B. (1979). Optimal Filtering. PrenticeHall, Englewood Cliffs, NJ.

[9] Li, P., Goodall, R. M., \& Kadirkamanathan, V. (2003). Parameter estimation of railway vehicle dynamic model using Rao-Blackwellised particle filter. In Proceedings of the 7th European Control Conference 2003, Cambridge, UK.

[10] Schön, T., \& Gustafsson, F. (2003). Particle filters for system identification of state-space models linear in either parameters or states. In Proceedings of the 13th IFAC Symposium on System Identification, pp1287-1292, Rotterdam, The Netherlands.

[11] Schön, T., Gustafsson, F., \& Nordlund, P-J. (2005). Marginalized Particle Filters for Mixed Linear/Nonlinear State-space Models. IEEE Trans. on Signal Processing, 53, pp2279-2289.

[12] Gordon, N. J., Salmond, D. J., \& Smith, A. F. M. (1993). Novel approach to nonlinear/non-Gaussian Bayesian state estimation. IEE Proceedings- $F$, 140(2): 107-113.

[13] Kitagawa, G. (1996). Monte Carlo filter and smoother for nonGaussian nonlinear state space models. Journal of Computational and Graphical Statistics, 5(1): 1-25.

[14] Doucet, A., Godsill, S., \& Ardrieu, C. (2000). On sequential Monte Carlo sampling methods for Bayesian filtering, Statistics and computing, 10:pp197-208.

[15] Bolviken, E., Acklam, P. J., Christophersen, N., \& Stordal, J-M. (2001). Monte Carlo filters for non-linear state estimation, Automatica, 37(2): pp177-183.

[16] Doucet, A., Freitas, N.de, \& Gordon, N. editors. (2001). Sequential Monte Carlo Methods in Practice. Statistics for Engineering and Information Science. Springer-Verlag, New York.

[17] Arulampalam, M. S., Maskell, S., Gordon, N., \& Clapp, T. (2002). A tutorial on particle filters for online nonlinear/non-Gaussian Bayesian tracking. IEEE Trans. on Signal Processing, 50, pp174-188.

[18] Kitagawa, G. (1998). A self-organizing state-space model. Journal of the American Statistical Association, 93(443): 1203-1215.

[19] Liu, J., \& West, M. (2001). Combined parameter and state estimation in simulation-based filtering. In Sequential Monte Carlo Methods in Practice, Doucet, A. Freitas, N.de and Gordon, N. editors. Statistics for Engineering and Information Science. Springer-Verlag, New York.

[20] Ardrieu, C., Doucet, A., Singh, S.S., \& Tadic, V.B. (2004). Particle methods for change detection, system identification and control. Proc. IEEE, 92(3) pp423-438.

[21] Chen, R. T. N., \& Tischler, M. B., (1987) The role of modelling and flight testing in rotorcraft parameter identification. Vertica, 11(4), pp619-647.

[22] Li, P., Postlethwaite, I., \& Turner, M. (2007). Subspace-based system identification for helicopter dynamic modelling. In Proceedings of the 63rd Annual Forum of the American Helicopter Society, Virginia, USA. 